ИЗВЕСТИЯ АКАДЕМИИ НАУК ЭСТОНСКОИ ССР. ТОМ 23 ХИМИЯ * ГЕОЛОГИЯ. 1974, 수 2

\title{
ХИМИЧЕСКИЙ СОСТАВ ПОДЗЕМНЫХ ВОД КАК ИНДИКАТОР ВОДОУПОРНЫХ СВОЙТВ КАРБОНАТНЫХ ПОРОД
}

Существующие в толщах пород водоносные горизонты могут объединять различные или одинаковые по составу подземные воды. Сходство составов может быть обусловлено или совпадением химико-физических условий (в том числе одинаковым химическим составом водовмещающих пород), или интенсивным водообменом между водоносными горизонтами.

Обратим вниманне на вторую причину. Гидрохимические данные для корреляции водоносных горизонтов впервые, по-видимому, применены гидрогеологами Камского филиала ВНИГНИ (Шуробор и др., 1970), которые использовали для этого величины общей минерализации, т. е. отношения ионов $\mathrm{SO}_{4}{ }^{\prime \prime} / \mathrm{Cl}^{\prime}$ и $\mathrm{Na}^{\circ} \mathrm{Cl}^{\prime}$ и содержание микрокомпонентов. В условиях карбонатных пород ордовика и силура Северной Эстонии разнообразие типов химического состава подземных вод можно использовать в целях распознавания водоносных горизонтов и водоупоров.

Основной осложняющий фактор при этом - неравномерная вскрытость по разрезу водовмещающих пород опробованными на воду буровыми скважинами, вследствие чего извлекаемая вода оказывается смешанной, ее химический состав представляет собой средневзвешенный состав подземных вод вскрытых водоносных горизонтов (слоев) и определяется элементарной формулой

$$
c_{r k}=\frac{Q_{1 r} c_{1 h}+Q_{2 r} c_{2 k}+\ldots+Q_{N r} c_{N k}}{Q_{1 r}+Q_{2 r}+\ldots+Q_{N r}}=\frac{\sum_{i=1}^{N} Q_{i r} c_{i k}}{\sum_{i=1}^{N} Q_{i}}
$$

Здесь $c_{r k}$

$$
\begin{aligned}
& c_{1 k}, c_{2 k}, \ldots, c_{N k}- \\
& Q_{1 r}, Q_{2 r}, \ldots, Q_{N r}-
\end{aligned}
$$

ких водоносных горизонтов. равен

$$
Q_{i r}=\frac{2,73 k_{i} m_{i r} S_{i r}}{\log \frac{R_{i}}{r}}
$$


где $k_{1}, k_{2}, \ldots, k_{N} \quad-$ коэффициенты фильтрации пород $i$-го водоносного горизонта;

$m_{1 r}, m_{2 r}, \ldots, m_{N r}-$ мощности вскрытых частей $i$-го водоносного горизонта в скважине $r$;

$S_{1 r}, S_{2 r}, \ldots, S_{N r}$ - истинные понижения уровня воды в $i$-м водоносном горизонте;

$R_{1}, R_{2}, \ldots, R_{N}$ - радиусы влияния $i$-го водоносного горизонта; $r$ - радиус скважины.

Чтобы в (1) определить средние концентрации $k$ ионов каждого водоносного горизонта $c_{i k}=c_{1 k}, c_{2 k}, \ldots, c_{N k}$, требуется наличие достаточного количества скважин с соответствующими данными о дебитах и химических составах смешанных вод. Система уравнений, подлежащих решению, состоит из $R$ отнотипных уравнений вида (1). Искомые концентрации предлагаем вычислять по формуле

$$
c_{i k}=\sum_{r=1}^{R} \frac{p_{i r}}{\sum_{r=1}^{R} p_{i r}} c_{r k} .
$$

Здесь $p_{i r}=\frac{Q_{i r}}{\sum_{i=1}^{N} Q_{i r}}=\frac{k_{i} m_{i r} S_{i r}}{\log \frac{R_{i}}{r} \sum_{i=1}^{N} \frac{k_{i} m_{i r} S_{i r}}{\log \frac{R_{i}}{r}}}$

Определив величину водопроводимости $i$-го водоносного горизонта в пределах скважины $r$ по формуле

$$
T_{i r}=k_{i} m_{i r}
$$

и допустив, что радиусы влияния $R_{i}(i=1,2, \ldots, N)$ и, тем более, выражения $\log \frac{R_{i}}{r}$ мало отличаются друг от друга, преобразуем (4) к виду

$$
p_{i r}=\frac{T_{i r} S_{i r}}{\sum_{i=1}^{N} T_{i r} S_{i r}} .
$$

Целесообразность и обоснованность формул типа (3), определяющих значения параметров, показана ранее (Йгар, 1972).

По определению Д. Агапьева (1958), истинное понижение уровня воды в каждом водоносном горизонте равно

$$
S_{i r}=S_{r}-N_{i r}
$$

где $S_{r}$ - суммарное понижение уровня (разница между суммарным статическим и динамическим уровнями) в скважине $r$;

$N_{\text {ir }}$ - величина снижения или повышения пьезометрического уровня $h_{i} i$-го горизонта по отношению к суммарному уровню $h_{r}$ в скважине $r$.

Следовательно, отношение $p_{\text {ir }}$ зависит не только от абсолютных величин суммарных понижений $S_{r}$, но и главным образом от глубины залегания отдельных пьезометрических уровней $h_{i}$ относительно $h_{r}$. 
Отметим еще, что величину $S_{i r}$ можно не учитывать, если суммарное понижение $S_{r}$ достаточно велико и если пьезометрические уровни каждого водоносного горизонта $h_{i}$ располагаются близко друг к другу. В таком случае значение

$$
p_{i r}=\frac{T_{i r}}{\sum_{i=1}^{N} T_{i r}}
$$

мало отличается от значения $p_{i r}$ по формуле (6). Для оценки сходства двух составов вод можно пользоваться понятием расстояния. Сходство может выражаться как пифагорова мера расстояний между двумя точками в $k$-мерном пространстве (Шоу, 1968)

$$
d_{i j}=\sqrt{\left(c_{i 1}-c_{j 1}\right)^{2}+\left(c_{i 2}-c_{j 2}\right)^{2}+\ldots+\left(c_{i k}-c_{j k}\right)^{2}},
$$

где $c_{i 1}, c_{i 2}, \ldots, c_{i k}$ и $c_{j 1}, c_{i 2}, \ldots, c_{j k}-$ химический состав вод водоносных горизонтов соответственно номера $i$ и $j$.

Среднее расстояние в предделах одной группы вод, т. е. среднее внутригрупповое расстояние

$$
\bar{d}_{S}=\frac{\sum_{i=1}^{L} d_{i j}}{L},
$$

должно быть меньше средних расстояний между группами вод, определяемых равенством

$$
\bar{d}_{V}=\frac{\sum_{i=L+1}^{M} d_{i j}}{M-L} .
$$

При этом Х. Харман (Harman, 1960) в качестве признака группы рассматривает скачкообразное увеличение среднего внутригруппового расстояния в том случае, если в группу вовлечены члены посторонних групп, хотя фактически группа исчерпана.

Ч исленны й пример. Вычисления выполнены на основе данных 8 эксплуатационных скважин, расположенных в пос. Пээтри и его окрестностях (Пайдеский район ЭССР). Для расчетов из предыдущей работы автора (Иыгар, 1973) взяты величины водопроводимости $T_{i r}$, суммарных статических уровней $h_{r}$ и средние по скважинам высоты пьезометрических уровней $h_{i}$ (табл. 1). Матрица расстояний $d_{i j}$, определенных при помощи формулы (9) по средним концентрациям ионов $c_{i k}$ (табл. 2), показывает наличие нескольких различных типов подземных вод:

\begin{tabular}{l|rrrr} 
& $\mathrm{S}_{1} \mathrm{tm}$ & $\mathrm{S}_{1} \mathrm{jr}$ & $\mathrm{O}_{3} \mathrm{pk}$ & $\mathrm{O}_{3} \mathrm{pgA}$ \\
\cline { 2 - 5 } $\mathrm{S}_{1} \mathrm{jr}$ & 23,97 & & & \\
$\mathrm{O}_{3}$ pk & 20,32 & 14,76 & & \\
$\mathrm{O}_{3}$ pgA & 24,34 & 19,56 & 5,67 & \\
$\mathrm{O}_{3}$ pgM & 23,12 & 8,44 & 7,16 & 11,42
\end{tabular}

Как видно, воды тамсалуского $\left(\mathrm{S}_{1} \mathrm{tm}\right)$ и юуруского $\left(\mathrm{S}_{1} \mathrm{jr}\right)$ горизонтов значительно отличаются от вод нижележащих горизонтов (среднее $\bar{d}_{V}$ соответственно 22,94 и 14,25 ма.экв. \%). И, наоборот, воды поркуниского 


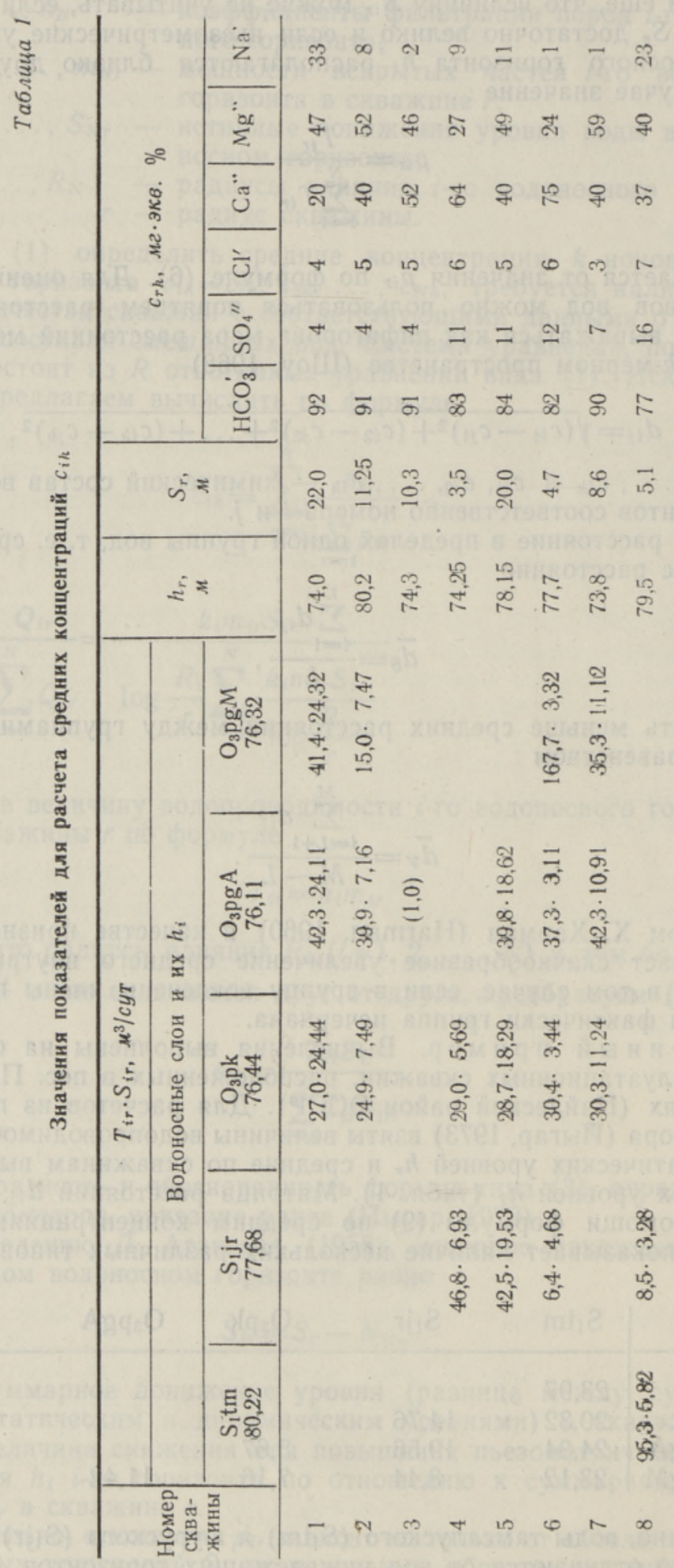




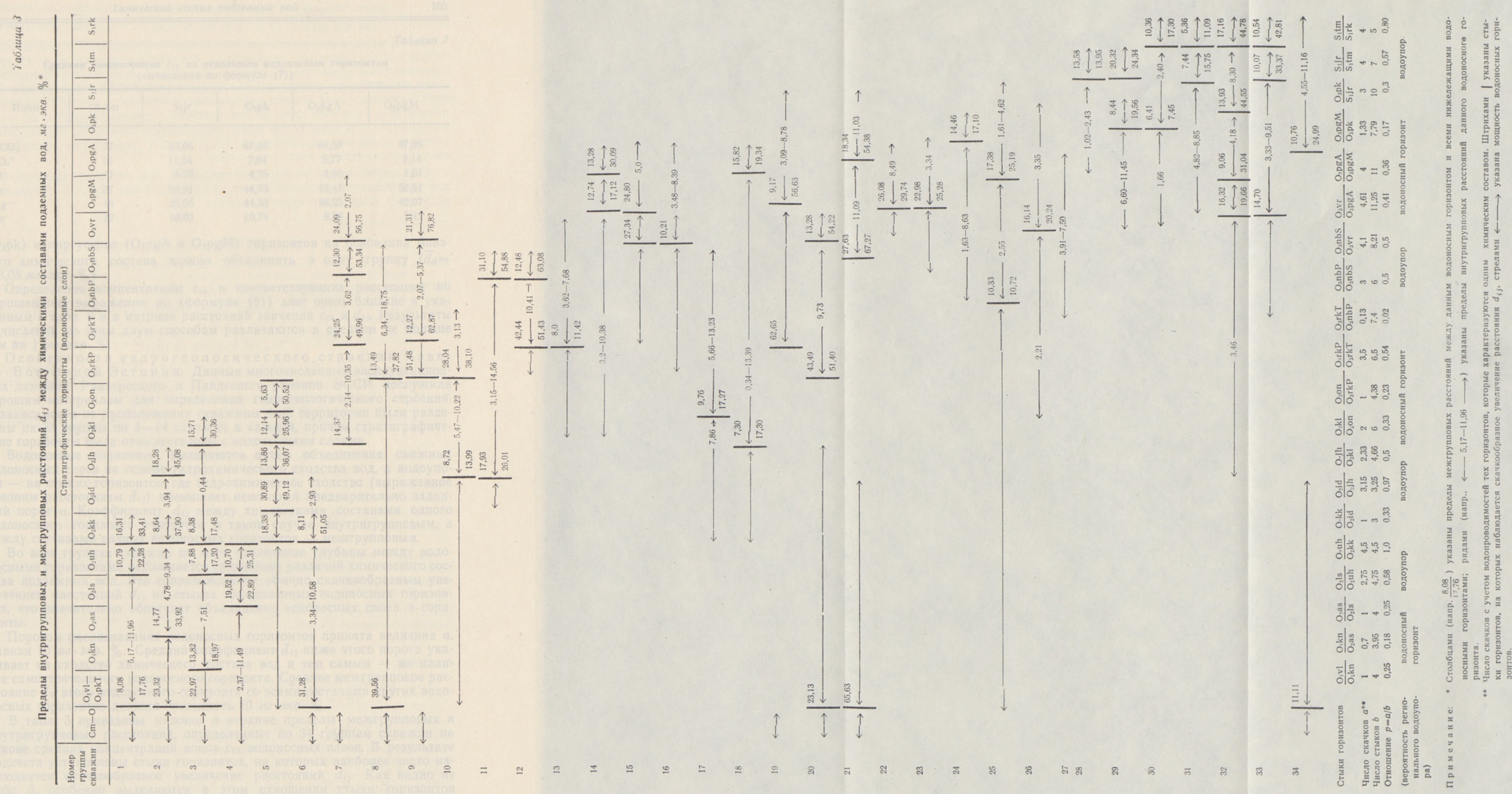


Средняя концентрация $c_{i k}$ по отдельным водоносным горизонтам (вычисления по формуле (7))

\begin{tabular}{l|r|r|r|r|r}
\hline \multicolumn{1}{c|}{ Ионы } & $\mathrm{S}_{1} \mathrm{tm}$ & $\mathrm{S}_{1} \mathrm{jr}$ & $\mathrm{O}_{3} \mathrm{pk}$ & $\mathrm{O}_{3} \mathrm{pgA}$ & $\mathrm{O}_{3} \mathrm{pg} M$ \\
\hline $\mathrm{HCO}_{3}{ }^{*}$ & 77 & 83,06 & 87,48 & 89,57 & 87,05 \\
$\mathrm{SO}_{4}{ }^{\prime}$ & 16 & 11,24 & 7,84 & 5,77 & 8,14 \\
$\mathrm{Cl}^{\prime}$ & 7 & 5,70 & 4,75 & 4,66 & 4,81 \\
$\mathrm{Ca}{ }^{*}$ & 37 & 54,91 & 44,93 & 43,41 & 50,51 \\
$\mathrm{Mg} \cdot$ & 40 & 35,06 & 44,33 & 48,25 & 40,07 \\
$\mathrm{Na} \cdot$ & 23 & 10,03 & 10,74 & 8,34 & 9,42
\end{tabular}

$\left(\mathrm{O}_{3} \mathrm{pk}\right)$ и пиргуского $\left(\mathrm{O}_{3} \mathrm{pgA}\right.$ и $\left.\mathrm{O}_{3} \mathrm{pgM}\right)$ горизонтов на основании близкого химического состава можно объединить в одну группу $\left(\bar{d}_{\boldsymbol{S}}=\right.$ $=8,08$ мг.экв. \%).

Определение концентрации $c_{i k}$ и соответствующих расстояний по упрощенному выражению $p_{i r}$ (формула (8)) дает очень близкие к указанным в таб́л. 2 и в матрице расстояний значения $c_{i k}$ и $d_{i j}$. Результаты вычислений по этим двум способам различаются в среднем не больше чем на 1 мг.экв. \%.

Особенности гидрогеологического строения Севе ро - В осточн ой $Э$ с тон и и. Данные многочисленных эксплуатационных скважин Раквереского и Пайдеского районов ЭССР послужили хорошим материалом для определения гидрогеологического строения. В зависимости от расположения скважины этой территории были разделены на 34 группы по 4-14 скважин в каждой, причем стратиграфические горизонты были отождествлены с водоносными слоями.

Водоносные горизонты выделяются путем объединения смежных водоносных слоев на основе гидрохимического сходства вод, а водоупоры - на стыках горизонтов, где гидрохимическое сходство (выраженное оредним расстоянием $\bar{d}_{i j}$ ) превышает некоторый предварительно заданный порог $\alpha$. Коэффициент $d_{i j}$ между химическими составами одного водоносного горизонта считается в таком случае внутригрупповым, а между составами разных водоносных горизонтов - межгрупповым.

Во всех группах скважин по мере увеличения глубины между водоносными горизонтами наблюдается увеличение различий химического состава подземных вод. Это сопровождается обычно скачкообразным увеличением расстояний $d_{i j}$ на стыках определенных водоносных горизонтов, что значительно облегчает объединение водоносных слоев в горизонты.

Порогом распознавания водоносных горизонтов принята величина $\alpha$, равная 10 мг экв. \%. Средний коэффициент $\bar{d}_{i j}$ ниже этого порога указывает на сходство химического состава вод и тем самым - на наличие самостоятельного водоносного горизонта. Среднее межгрупповое расстояние $\bar{d}_{V}$ этого водоносного горизонта со всеми составами других водоносных горизонтов должнб превышать 10 мә.экв. \%.

В табл. 3 приведены нижние и верхние пределы межгрупповых и внутригрупповых расстояний, определенные по 34 группам скважин на основе средних концентраций ионов $c_{i k}$ водоносных слоев. В результате подсчета установлены стыки горизонтов, на которых наиболее часто наблюдается скачкообразное увеличение расстояний $d_{i j}$. Как видно из табл. 3, особенно выделяются в этом отношении стыки горизонтов $\mathrm{O}_{2} \mathrm{uh} / \mathrm{O}_{2} \mathrm{kk}, \mathrm{O}_{2} \mathrm{id} / \mathrm{O}_{2} \mathrm{jh}, \mathrm{O}_{3} \mathrm{nbP} / \mathrm{O}_{3} \mathrm{nbS}, \mathrm{O}_{3} \mathrm{nbS} / \mathrm{O}_{3} \mathrm{vr}$ и $\mathrm{S}_{1} \mathrm{tm} / \mathrm{S}_{1} \mathrm{rk}$. 
Скачкообразное увеличение расстояний обусловлено прежде всего типовыми изменениями химнческого состава подземных вод, которые сохраняются в природных условиях благодаря водоупорам. Региональными водоупорами в северной части рассматриваемой территории являются диктионемовый сланец пакерортского горизонта ( $\left.\mathrm{O}_{1} \mathrm{pkT}\right)$, промышленная пачка горючих сланцев кукрузеского горизонта $\left(\mathrm{O}_{2} \mathrm{kk}\right)$ и, по-видимому, мергели и известняки всего идавереского горизонта $\left(\mathrm{O}_{2} \mathrm{id}\right)$. Водоупорные свойства карбонатных пород других горизонтов ордовика и силура проявляются лишь участками.

Описанный метод можно рекомендовать как наиболее эффективный для установления гидрогеологического строения при помоши неполных и косвенных гидрогеологических данных.

\section{ЛИТЕРАТ У Р А}

Агапьев Д. Ф. 1958. Определение фильтращионных свойств пород путем суммарного опробования водоносных горизонтов. Разведка и охрана недр, № 11.

П ы гар П. 1972. Вычисление параметров водопроницаемости коренных пород. Изв. АН ЭССР, Хим. Геол., 21, № 4.

Иы га р П. 1973. Определение гидрогеологического строения при помощи суммарных уровней подземных вод. Изв. АН ЭССР, Хим. Геол., 22, № 4.

Шоу Д. М. 1968. О делении данных в аналитической геохимии на две группы с помощью дистанционного коэффициента. В сб.: Вопросы математической геологии. Л.

Шуробор Ю. В., Шуробор А. В., Шестов И. Н. 1970. Использование приемов математической статистики для корреляции водоносных горизонтов по гидрохимическим данным. Тр. Камского фнлиала ВНИГНИ, вып. 72.

$\mathrm{H}$ a r m a n H. H. 1960. Modern factor analysis. Univ. Chicago Press.
Ннститут геологии
Академии наук Эстонской ССР
Поступила в редакцию
$12 / \mathrm{X}$
1973

\section{P. JOGAR}

\section{POHJAVETE KEEMILINE KOOSTIS KUI VETTPIDAVATE KIVIMITE INDIKAATOR}

Artiklis käsitletakse vett sisaldavate kihtide stratifitseerimist summaarse, s. o. mitmest kihist pumbatava põhjavee keemilise koostise abil. Oksikute kihtide põhjavete iga iooni keskmine kontsentratsioon $c_{i k}$ arvutatakse suhtelise tōenäosuse alusel (7), kus $r=1$, $2, \ldots, R$ (puuraukude arv) $i=1,2, \ldots, N$ (lademete arv) ja $p_{\text {ir }}-i$-nda lademe veejuhtivuse osakaal $r$-ndas puuraugus. Vett sisaldavate kihtide veehorisontideks ühendamiseks kasutatakse keskmiste keemiliste koostiste omavahelisi kaugusi. Analüüsiks kasutati Kirde-Eestis asuva 34 puuraukude grupi kohta saadud andmeid (tab. 3).

\section{P. JOGAR}

\section{THE CHEMICAL COMPOSITION OF UNDERGROUND WATERS AS AN INDICATOR OF WATER-RESISTANT ROCKS}

The author discusses the stratification of water-containing strata with the help of the chemical composition of summary underground waters, i.e. of those pumped from different strata. The mean concentration $c_{i k}$ of each ion of single strata is calculated on the basis of relative probability (7), where $r=1,2, \ldots, R$ (number of boreholes), $t=1,2, \ldots, N$ (number of strata), $p_{i r}$ - the partial weight share of the water conductivity of the $i$-th stratum in the $r$-th borehole. For uniting the water-containing strata into water horizons, the mutual distances of the mean chemical compositions are used. For analysis, the data on a group of 34 boreholes of North-east Estonia were used (Table 3). 